\title{
Uniqueness, Born Approximation, and Numerical Methods for Diffuse Optical Tomography
}

\author{
Kiwoon Kwon \\ Department of Mathematics, Dongguk University, Seoul 100-715, Republic of Korea \\ Correspondence should be addressed to Kiwoon Kwon; kwkwon@dongguk.edu
}

Received 8 April 2013; Accepted 12 May 2013

Academic Editor: Chang-Hwan Im

Copyright (C) 2013 Kiwoon Kwon. This is an open access article distributed under the Creative Commons Attribution License, which permits unrestricted use, distribution, and reproduction in any medium, provided the original work is properly cited.

Diffuse optical tomogrpahy (DOT) is to find optical coefficients of tissue using near infrared light. DOT as an inverse problem is described and the studies about unique determination of optical coefficients are summarized. If a priori information of the optical coefficient is known, DOT is reformulated to find a perturbation of the optical coefficients inverting the Born expansion which is an infinite series expansion with respect to the perturbation and the a priori information. Numerical methods for DOT are explained as methods inverting first- or second-order Born approximation or the Born expansion itself.

\section{Introduction}

DOT is to find optical coefficients of tissue using near infrared light. DOT is known to be of low cost, portable, nonionized, and nonmagnetized. And DOT has higher temporal resolution and more functional information than conventional structural medical imaging modalities such as magnetic resonance imaging (MRI) and computerized tomography (CT). For the comparison to other functional imaging modalities such as functional MRI (fMRI), photon emission tomography (PET), and electroencephalogram (EEG), see [1] . DOT is used in the area of breast imaging [2-4], functional neuroimaging $[5,6]$, brain computer interface $(\mathrm{BCI})[7,8]$, and the study about seizure $[9,10]$, newborn infants $[11,12]$, osteoarthritis [13], and rat brain $[14,15]$.

In this paper, DOT is explained as an inverse problem with respect to a forward problem formulated as an elliptic partial differential equation. Propagation of light in biological tissues is usually described by diffusion approximation equation in the frequency domain, the simplest but nontrivial approximation of the Boltzmann equation, as follows:

$$
\begin{gathered}
-\nabla \cdot(\kappa \nabla \Phi)+\left(\mu_{a}+\frac{i \omega}{c}\right) \Phi=q \quad \text { in } \Omega, \\
\Phi+2 a \nu \cdot(\kappa \nabla \Phi)=0 \quad \text { on } \partial \Omega,
\end{gathered}
$$

where $v$ is an outer unit normal vector, $\Phi$ is a photon density distribution, $\kappa=1 / 3\left(\mu_{a}+\mu_{s}^{\prime}\right)$ is an diffusion coefficient, $\mu_{a}$ is an absorption coefficient, $\mu_{s}^{\prime}$ is a reduced scattering coefficient, and $a$ is a reflection coefficient.

If $\partial \kappa / \partial \nu=0$ on $\partial \Omega$, by setting $\Psi=\sqrt{\kappa} \Phi$ and $k=$ $\sqrt{\Delta \sqrt{\kappa} / \sqrt{\kappa}+\mu_{a} / \kappa+i(\omega / \mathcal{L})}$ with $\operatorname{Im}(k) \geq 0$, we have

$$
\begin{gathered}
-\Delta \Psi+k^{2} \Psi=\frac{q}{\sqrt{\kappa}} \quad \text { in } \Omega, \\
\Psi+2 a \nu \cdot(\kappa \nabla \Psi)=0 \quad \text { on } \partial \Omega .
\end{gathered}
$$

If $k$ is constant and $q(\cdot) / \sqrt{\kappa}=\delta\left(\cdot, r_{s}\right)$ for some source point $r_{s}$, we have the following solution of $(2 \mathrm{a})$ :

$$
\Psi(r)=R\left(r, r_{s}\right)=\frac{e^{i k\left|r-r_{s}\right|}}{4 \pi\left|r-r_{s}\right|} .
$$

DOT is to find the optical coefficients $\mu_{a}$ and/or $\mu_{s}^{\prime}$ from the measurement information $\Phi_{i, j}$ which is the value of the solution of (1a) and (1b) at $r_{i} \in \partial \Omega$ when $q(r)=\delta\left(r, r_{j}\right), r_{j} \in$ $\partial \Omega$. The $r_{i}$ and $r_{j}$ are usually called source and detector point, respectively.

In Section 2, the unique determination of the optical coefficients is discussed and many known results are summarized for the uniqueness questions. In Section 3, DOT is 
reformulated as to find perturbation of the optical coefficient inverting the Born expansion. The errors of the Born approximation in the Lebesgue and Sobolev norms are given. In Section 4, numerical methods of DOT are mainly described as the methods inverting the first-, second-, and higher-order Born approximation and Born expansion itself.

\section{Uniqueness}

The research about unique determination of the optical coefficients in DOT is rare except [16], but it is a very important issue for DOT as an inverse problem. The determinaiton of optical coefficients $\left(\mu_{a}, \mu_{s}\right)$ in (1a) and (1b) is equivalent to the determination of $k$ in (2a) and (2b) when $\omega \neq 0$.

When $\mu_{a}, \kappa, \nabla \kappa$ have upper and lower bound and $q$ is contained in $H^{-1}(\Omega)$ or a Dirac delta function, (1a) and (1b) have a unique solution $\Phi \in H^{1}(\Omega)$ and (2a) and (2b) have a unique solution $\Psi \in H^{1}(\Omega)[17,18]$.

Boundary value problem (1a) and (1b) with $q(r)=\delta\left(r, r_{s}\right)$ is equivalent to boundary value problem with (1a) for $q=0$ and nonzero Robin boundary condition replacing (1b). This argument can be proved using the function $H$ in [19]. Therefore, DOT is redescribed as to find the optical coefficients from the Robin-to-Dirichlet map defined as a map from $H^{-1 / 2}(\partial \Omega)$ to $H^{1 / 2}(\partial \Omega)$. Using unique solvability of (1a) with the Dirichlet or Neumann boundary condition replacing (1b), the Robin-to-Dirichlet map is equivalent to the Neumann-toDirichlet map and to the Dirichlet-to-Neumann map. Since $\operatorname{Im}(k)>0$ for $\omega \neq 0, k$ is not a Dirichlet eigenvalue of (2a). Therefore, knowing the Dirichlet-to-Neumann map is also equivalent to knowing farfield map in inverse scattering problem $[20,21]$.

Using above results and many known results for inverse scattering problem, we summarized the uniqueness and nonuniqueness results as follows.

Case $1\left(\kappa=1, \mu_{a}=1+(m-1) \chi_{D}, m \neq 1\right)$. The cases for positive constant $m$ can be understood as special cases of Case 2 . The limiting cases $m=\infty$ and $m=0$ could be considered as (la) in $\Omega \backslash \bar{D}$ with the Robin boundary condition (lb) on $\partial \Omega$ and the boundary condition on $\partial D$ as $\Phi=0$ (sound-soft case) and $\partial \Phi / \partial \nu=0$ (sound-hard case) on $\partial D$. The uniqueness for sound-soft and sound-hard obstacle $D$ is considered in $[20,22]$.

Case $2\left(\kappa=1, \mu_{a}=1+(m(x)-1) \chi_{D}\right.$, where $m(x) \neq 1$ on $\partial D)$. This case is called "inverse transmission problem" and the uniqueness is solved in [20].

Case $3\left(\kappa=1, \mu_{a}=\mu_{a}(x)\right)$. In [16], nonuniqueness of $\mu_{a}(x)$ is shown by assuming that refractive index is not determined or we use only continuous wave light source. However, if refractive index is known and $\omega \neq 0$, unique determination of the optical coefficient is possible. This nonuniqueness is proved by using [23].

Case $4\left(\kappa=I_{n}+\left(K(x)-I_{n}\right) \chi_{D}, \mu_{a}=1+(m(x)-1) \chi_{D}\right)$. Here, $K(x)$ is a unknown positive-definite matrix function such that $K(x) \neq I_{n}$ on $\partial D$ and $m(x)$ is a positive function such that $m(x) \neq 1$ on $\partial D$. The uniqueness of $D$ is solved in $[21,24-28]$ and the nonuniqueness of $K(x)$ is reported in [24, $29,30]$. Therefore, although the domain of nonhomogenity $D$ can be uniquely determined by infinite measurements, the nonhomogeneous anisotropic diffusion coefficient $K(x)$ cannot be determined uniquely. Similar results are known for the nonuniqueness and illusion for anisotropic nonhomogeneous electric conductivity [24, 31].

\section{Born Approximation}

Suppose that we know a priori information $x^{0}=\left(\kappa^{0}, \mu_{a}^{0}\right)$ about the optical coefficients $x=\left(\kappa, \mu_{a}\right)$ to find. Then, DOT is redescribed as to find the perturbation $\delta x=x-x^{0}=$ $\left(\delta \kappa, \delta \mu_{a}\right)$.

Let $\Phi$ and $\Phi^{0}$ be the solutions of (1a) and (1b) for $x$ and $x^{0}$, respectively. When $q(r)=\delta\left(r, r_{s}\right), \Phi$ and $\Phi^{0}$ are the Robin function $R\left(\cdot, r_{s}\right)$ and $R^{0}\left(\cdot, r_{s}\right)$ for the optical coefficients $x$ and $x^{0}$, respectively. $R\left(\cdot, r_{s}\right)$ is expanded as the Born expansion, an infinite series, with respect to $R^{0}\left(\cdot, r_{s}\right)$ and $\delta x$ as follows:

$$
R\left(r, r_{s}\right)=R^{0}\left(r, r_{s}\right)+\mathscr{R} R^{0}\left(\cdot, r_{s}\right)+\mathscr{R}^{2} R^{0}\left(\cdot, r_{s}\right)+\cdots,
$$

where

$$
\begin{aligned}
(\mathscr{R} \Psi)(r)=\mathscr{R}(\delta x) \Psi(r)=\left(\mathscr{R}_{1} \Psi\right)(r)+\left(\mathscr{R}_{2} \Psi\right)(r), & \\
\left(\mathscr{R}_{1} \Psi\right)(r) & =\mathscr{R}_{1}\left(\delta \mu_{a}\right) \Psi(r) \\
& =-\int_{\Omega} \delta \mu_{a}\left(r^{\prime}\right) R\left(r, r^{\prime}\right) \Psi\left(r^{\prime}\right) d r^{\prime}, \\
\left(\mathscr{R}_{2} \Psi\right)(r) & =\mathscr{R}_{2}(\delta \kappa) \Psi(r) \\
& =-\int_{\Omega} \delta \kappa\left(r^{\prime}\right) \nabla R\left(r, r^{\prime}\right) \nabla \Psi\left(r^{\prime}\right) d r^{\prime} .
\end{aligned}
$$

It is proved that the $n$th order term in the above Born expansion (4) is the $n$th order Frechet derivative divided by $n$ ! [18]. The bounds for the operators $\mathscr{R}_{1}$ and $\mathscr{R}_{2}$ are given in the Sobolev, Lebesque, and weighted Sobolev spaces norms [18]. The estimate for the Lebesque space norms for $\mathscr{R}_{1}$ is also given in [32]. Therefore, there exist positive constants $C_{1}$ and $\mathrm{C}_{2}$ such that

$$
\begin{gathered}
\left\|\mathscr{R}_{1}\right\|_{w^{1, p}(\Omega) \rightarrow w^{1, p}(\Omega)} \leq C_{1}\left\|\delta \mu_{a}\right\|_{L^{\infty}(\Omega)}, \\
\left\|\mathscr{R}_{2}\right\|_{w^{1, p}(\Omega) \rightarrow w^{1, p}(\Omega)} \leq C_{2}\|\delta \kappa\|_{L^{\infty}(\Omega)},
\end{gathered}
$$

for $p \geq 1$. The detailed estimate for the coefficients $C_{1}, C_{2}$ in special cases is given in $[18,32]$.

If

$$
C_{3}=C_{1}\left\|\delta \mu_{a}\right\|_{\infty}+C_{2}\|\delta \kappa\|_{\infty}<1
$$

holds, then the error of the $m$ th order Born approximation $B^{m} R^{0}\left(\cdot, r_{s}\right)$ is given by

$$
\left\|R\left(\cdot, r_{s}\right)-B^{m} R^{0}\left(\cdot, r_{s}\right)\right\|_{W^{1, p}} \leq C_{3}^{m}\left\|R\left(\cdot, r_{s}\right)\right\|_{W^{1, p}(\Omega)}
$$

using the estimates (6a) and (6b). 


\section{Numerical Methods}

Numerical methods for DOT are explained in terms of solving the Born approximation given in Section 3. Other numerical methods not categorized as inverting Born approximation are commented in the final section.

4.1. Linearized Methods. Linearized DOT to find $\delta x$ by solving the first-order Born approximation is studied by many researchers in the initial stage of DOT. Although this method lacks exact recovery in most cases, it is used still frequently when faster real-time computing is needed and very good a priori information is given. In this method, the discretized problem is an algebraic equation and it is essential to use efficient matrix solver. In the Jacobian matrix, the number of rows is the number of measurements and the number of columns is the number of elements to be determined. In most cases, the number of elements is larger than the number of measurements in order to obtain higher resolution image. Therefore, the algebraic equation is usually an underdetermined system. Efficient matrix solvers including arithmetic reconstruction technique (ART), simultaneous arithmetic reconstruction technique (SART), simultaneous iterative reconstruction technique (SIRT), and the Krylov space methods are studied [33, 34]. Since the linearized problems also have ill-posed property of DOT, there are many researches about regularization methods including the Tikhonov regularization. The dependence on the discretization error due to ill posedness of the linearized method is reported in [35].

4.2. Nonlinear Methods. Nonlinear method is to find $\delta x$ by solving the Born expansion [36]. This method is usually formulated as optimization problem and is solved by the Newton-type method including Levenberg-Marquardt method [37, 38]. A few softwares based on nonlinear method with finite element forward solver with corresponding references are as follows:

(i) TOAST (Time-Resolved Optical Absorption and Scattering Tomography) [39],

(ii) NIRFAST (Near InfraRed Florescence and Spectral Tomography) [40, 41],

(iii) PMI (Photon Migration Imaging) Toolbox [42].

Nonlinear method needs heavy computation due to large iteration numbers. To reduce the heavy computation, there are studies about efficient numerical techniques such as multigrid, domain decomposition [43], and adaptive [44] method.

4.3. Inverse Born Approximation of Order Higher Than Two. This method is to find $\delta x$ by solving second- and higher-order Born approximation. In fact, solving the Born approximation of order higher than two is implicit but can be approximated by explicit inverse Born approximation. Formal inverse of the Born approximation is called inverse Born approximation. The first-order inverse Born approximation corresponds to linearized DOT and inverse Born expansion itself corresponds to (nonlinear) DOT. Higher-order methods improve the order of convergence for lower-order methods. The error of the inverse Born approximation is given and analyzed in terms of the Lebesgue space norms when $\delta \kappa=0$ [45] and in terms of the Sobolev space norms for the second order [46].

4.4. Other Methods. The solution of (1a) and (1b) is usually solved by finite element method [36]. Another approach for the forward problem is to compute directly the Robin function using the Fourier-Laplace transform [47-50]. The disadvantage of this method is that it depends on the special geometry of the region of interest and the inverse FourierLaplace transfrom is known to be severely ill posed.

Equations (la) and (lb) could be replaced by probabilistic approach in the Monte Carlo method [51]. The method takes much more time than finite element method and highly depends on random number generator. The comparison of finite element method and the Monte Carlo method is done by many papers including [52].

The diffusion approximation (1a) and (lb) is the firstorder approximation of radiative transfer equation. There are studies about DOT based on radiative transfer equation $[53,54]$ and its $n$th order approximation $[55]$.

\section{Conclusion}

Unique determination of DOT is surveyed. The study about nonuniqueness for anisotropic diffusion coefficients and for unknown refractive index is also surveyed. The perturbation of photon density with respect to the perturbed optical coefficient is expanded using the Born expansion and the error analysis removing higher-order terms is given. The numerical methods for DOT are described by inverting first-, second-, and higher-order Born approximation, and the Born expansion itself is reviewed.

\section{Acknowledgment}

This work is supported by Basic Science Research Program through the National Research foundation of Korea (NRF) funded by the Ministry of Education (2010-0004047).

\section{References}

[1] B. White, Developing high-density diffuse optical tomography for neuroimaging [Ph.D. thesis], Washington University, St. Louis, Mo, USA, 2012.

[2] Q. Zhang, T. J. Brukilacchio, A. Li et al., "Coregistered tomographic X-ray and optical breast imaging: initial results," Journal of biomedical optics, vol. 10, no. 2, Article ID 024033, 2005.

[3] T. Durduran, R. Choe, G. Yu et al., "Diffuse optical measurement of blood flow in breast tumors," Optics Letters, vol. 30, no. 21, pp. 2915-2917, 2005.

[4] V. Ntziachristos, A. G. Yodh, M. Schnall, and B. Chance, "Concurrent MRI and diffuse optical tomography of breast after indocyanine green enhancement," Proceedings of the National Academy of Sciences of the United States of America, vol. 97, no. 6, pp. 2767-2772, 2000. 
[5] J. P. Culver, A. M. Siegel, M. A. Franceschini, J. B. Mandeville, and D. A. Boas, "Evidence that cerebral blood volume can provide brain activation maps with better spatial resolution than deoxygenated hemoglobin," NeuroImage, vol. 27, no. 4, pp. 947-959, 2005.

[6] M. A. Franceschini and D. A. Boas, "Noninvasive measurement of neuronal activity with near-infrared optical imaging," NeuroImage, vol. 21, no. 1, pp. 372-386, 2004.

[7] R. Sitaram, H. Zhang, C. Guan et al., "Temporal classification of multichannel near-infrared spectroscopy signals of motor imagery for developing a brain-computer interface," NeuroImage, vol. 34, no. 4, pp. 1416-1427, 2007.

[8] S. M. Coyle, T. E. Ward, and C. M. Markham, "Braincomputer interface using a simplified functional near-infrared spectroscopy system.," Journal of Neural Engineering, vol. 4, no. 3, pp. 219-226, 2007.

[9] E. Watanabe, A. Maki, F. Kawaguchi, Y. Yamashita, H. Koizumi, and Y. Mayanagi, "Noninvasive cerebral blood volume measurement during seizures using multichannel near infrared spectroscopic topography," Journal of Biomedical Optics, vol. 5, no. 3, pp. 287-290, 2000.

[10] K. Buchheim, H. Obrig, W. V. Pannwitz et al., "Decrease in haemoglobin oxygenation during absence seizures in adult humans," Neuroscience Letters, vol. 354, no. 2, pp. 119-122, 2004.

[11] G. Taga, Y. Konishi, A. Maki, T. Tachibana, M. Fujiwara, and H. Koizumi, "Spontaneous oscillation of oxy- and deoxyhemoglobin changes with a phase difference throughout the occipital cortex of newborn infants observed using noninvasive optical topography," Neuroscience Letters, vol. 282, no. 1-2, pp. 101-104, 2000.

[12] T. Austin, A. P. Gibson, G. Branco et al., "Three dimensional optical imaging of blood volume and oxygenation in the neonatal brain," NeuroImage, vol. 31, no. 4, pp. 1426-1433, 2006.

[13] Z. Yuan, Q. Zhang, E. Sobel, and H. Jiang, “Three-dimensional diffuse optical tomography of osteoarthritis: Initial results in the finger joints," Journal of Biomedical Optics, vol. 12, no. 3, Article ID 034001, 2007.

[14] A. M. Siegel, J. P. Culver, J. B. Mandeville, and D. A. Boas, "Temporal comparison of functional brain imaging with diffuse optical tomography and fMRI during rat forepaw stimulation," Physics in Medicine and Biology, vol. 48, no. 10, pp. 1391-1403, 2003.

[15] J. P. Culver, T. Durduran, D. Furuya, C. Cheung, J. H. Greenberg, and A. G. Yodh, "Diffuse optical tomography of cerebral blood flow, oxygenation, and metabolism in rat during focal ischemia," Journal of Cerebral Blood Flow and Metabolism, vol. 23, no. 8, pp. 911-924, 2003.

[16] S. R. Arridge and W. R. B. Lionheart, "Nonuniqueness in diffusion-based optical tomography," Optics Letters, vol. 23, no. 11, pp. 882-884, 1998.

[17] D. Gilbarg and N. S. Trudinger, Elliptic Partial Differential Equations of Second Order, vol. 224 of Grundlehren der Mathematischen Wissenschaften, Springer, Berlin, Germany, 2nd edition, 1983.

[18] K. Kwon and B. Yazıc1, "Born expansion and Fréchet derivatives in nonlinear diffuse optical tomography," Computers \& Mathematics with Applications, vol. 59, no. 11, pp. 3377-3397, 2010.

[19] C. Miranda, Partial Differential Equations of Elliptic Type, Ergebnisse der Mathematik und ihrer Grenzgebiete, Band 2, Springer, New York, NY, USA, 1970.
[20] D. Colton and R. Kress, Inverse Acoustic and Electromagnetic Scattering Theory, vol. 93 of Applied Mathematical Sciences, Springer, Berlin, Germany, 2nd edition, 1998.

[21] V. Isakov, "On uniqueness in the inverse transmission scattering problem," Communications in Partial Differential Equations, vol. 15, no. 11, pp. 1565-1587, 1990.

[22] A. Kirsch and R. Kress, "Uniqueness in inverse obstacle scattering," Inverse Problems, vol. 9, no. 2, pp. 285-299, 1993.

[23] J. Sylvester and G. Uhlmann, "A global uniqueness theorem for an inverse boundary value problem," Annals of Mathematics. Second Series, vol. 125, no. 1, pp. 153-169, 1987.

[24] K. Kwon, "Identification of anisotropic anomalous region in inverse problems," Inverse Problems, vol. 20, no. 4, pp. 1117-1136, 2004.

[25] K. Kwon and D. Sheen, "Anisotropic inverse conductivity and scattering problems," Inverse Problems, vol. 18, no. 3, pp. 745756, 2002.

[26] M. Piana, "On uniqueness for anisotropic inhomogeneous inverse scattering problems," Inverse Problems, vol. 14, no. 6, pp. 1565-1579, 1998.

[27] R. Potthast, "On a concept of uniqueness in inverse scattering from an orthotopic medium," Journal of Integral Equations and Applications, vol. 11, pp. 197-215, 1999.

[28] D. Sheen and D. Shepelsky, "Inverse scattering problem for a stratified anisotropic slab," Inverse Problems, vol. 15, no. 2, pp. 499-514, 1999.

[29] G. Eskin, "Inverse scattering problem in anisotropic media," Communications in Mathematical Physics, vol. 199, no. 2, pp. 471-491, 1998.

[30] F. Gylys-Colwell, "An inverse problem for the Helmholtz equation," Inverse Problems, vol. 12, no. 2, pp. 139-156, 1996.

[31] K. Kwon, "The uniquess, cloaking, and illusion for electrical impedance tomography," Submitted.

[32] S. Moskow and J. C. Schotland, "Convergence and stability of the inverse scattering series for diffuse waves," Inverse Problems, vol. 24, no. 6, Article ID 065005, 16 pages, 2008.

[33] S. R. Arridge and J. C. Schotland, "Optical tomography: forward and inverse problems," Inverse Problems, vol. 25, pp. 1-59, 2009.

[34] J. Kaipio and E. Somersalo, Statistical and Computational Inverse Problems, vol. 160 of Applied Mathematical Sciences, Springer, New York, NY, USA, 2005.

[35] M. Guven, B. Yazici, K. Kwon, E. Giladi, and X. Intes, "Effect of discretization error and adaptive mesh generation in diffuse optical absorption imaging. I," Inverse Problems, vol. 23, no. 3, pp. 1115-1133, 2007.

[36] S. R. Arridge, "Optical tomography in medical imaging," Inverse Problems, vol. 15, no. 2, pp. R41-R93, 1999.

[37] K. Levenberg, "A method for the solution of certain non-linear problems in least squares," Quarterly of Applied Mathematics, vol. 2, pp. 164-168, 1944.

[38] D. Marquadt, "An algorithm for least-squares estimation of nonlinear parameters," IEEE Transactions of Circuits and Systems, vol. 26, 1979.

[39] TOAST website, April 2013, http://web4.cs.ucl.ac.uk/research/ vis/toast/.

[40] NIRFAST webiste, April 2013, http://www.dartmouth.edu/nir/ nirfast/index.php.

[41] H. Dehghani, M. E. Eames, P. K. Yalavarthy et al., "Near infrared optical tomography using NIRFAST: algorithm for numerical 
model and image reconstruction," Communications in Numerical Methods in Engineering with Biomedical Applications, vol. 25, no. 6, pp. 711-732, 2009.

[42] PMI Toolbox website, April 2013, http://www.nmr.mgh.harvard.edu/PMI/toolbox/.

[43] K. Kwon, B. Yazıc1, and M. Guven, "Two-level domain decomposition methods for diffuse optical tomography," Inverse Problems, vol. 22, no. 5, pp. 1533-1559, 2006.

[44] M. Guven, B. Yazici, K. Kwon, E. Giladi, and X. Intes, "Effect of discretization error and adaptive mesh generation in diffuse optical absorption imaging. II," Inverse Problems, vol. 23, no. 3, pp. 1135-1160, 2007.

[45] S. Moskow and J. C. Schotland, "Numerical studies of the inverse Born series for diffuse waves," Inverse Problems, vol. 25, no. 9, Article ID 095007, 18 pages, 2009.

[46] K. Kwon, "The second-order Born approximation in diffuse optical tomography," Journal of Applied Mathematics, vol. 2012, Article ID 637209, 15 pages, 2012.

[47] V. A. Markel and J. C. Schotland, "Inverse problem in optical diffusion tomography. I. Fourier-Laplace inversion formulas," Journal of the Optical Society of America A. Optics, Image Science, and Vision, vol. 18, no. 6, pp. 1336-1347, 2001.

[48] V. A. Markel and J. C. Schotland, "Inverse problem in optical diffusion tomography. II. Role of boundary conditions," Journal of the Optical Society of America A: Optics and Image Science, and Vision, vol. 19, no. 3, pp. 558-566, 2002.

[49] V. A. Markel, V. Mital, and J. C. Schotland, "Inverse problem in optical diffusion tomography. III. Inversion formulas and singular-value decomposition," Journal of the Optical Society of America A: Optics and Image Science, and Vision, vol. 20, no. 5, pp. 890-902, 2003.

[50] V. A. Markel, J. A. O'Sullivan, and J. C. Schotland, "Inverse problem in optical diffusion tomography. IV. Nonlinear inversion formulas," Journal of the Optical Society of America A: Optics and Image Science, and Vision, vol. 20, no. 5, pp. 903-912, 2003.

[51] L. Wang, S. L. Jacques, and L. Zheng, "MCML-Monte Carlo modeling of light transport in multi-layered tissues," Computer Methods and Programs in Biomedicine, vol. 47, no. 2, pp. 131-146, 1995.

[52] K. Kwon, T. Son, K. J. Lee, and B. Jung, "Enhancement of light propagation depth in skin: Cross-validation of mathematical modeling methods," Lasers in Medical Science, vol. 24, no. 4, pp. 605-615, 2009.

[53] G. S. Abdoulaev and A. H. Hielscher, "Three-dimensional optical tomography with the equation of radiative transfer," Journal of Electronic Imaging, vol. 12, no. 4, pp. 594-601, 2003.

[54] T. Tarvainen, M. Vauhkonen, V. Kolehmainen, and J. P. Kaipio, "Finite element model for the coupled radiative transfer equation and diffusion approximation," International Journal for Numerical Methods in Engineering, vol. 65, no. 3, pp. 383405, 2006.

[55] E. D. Aydin, C. R. E. de Oliveira, and A. J. H. Goddard, "A compariosn between transport and diffusion calculations using a finite element-spherical harmonics ratiation tranport method," Medical Physics, vol. 2, pp. 2013-2023, 2002. 


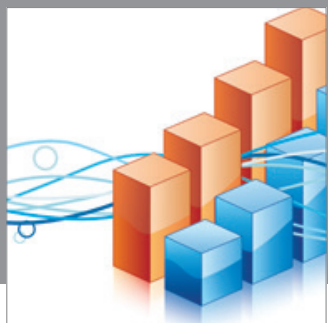

Advances in

Operations Research

mansans

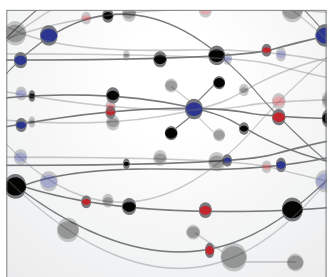

The Scientific World Journal
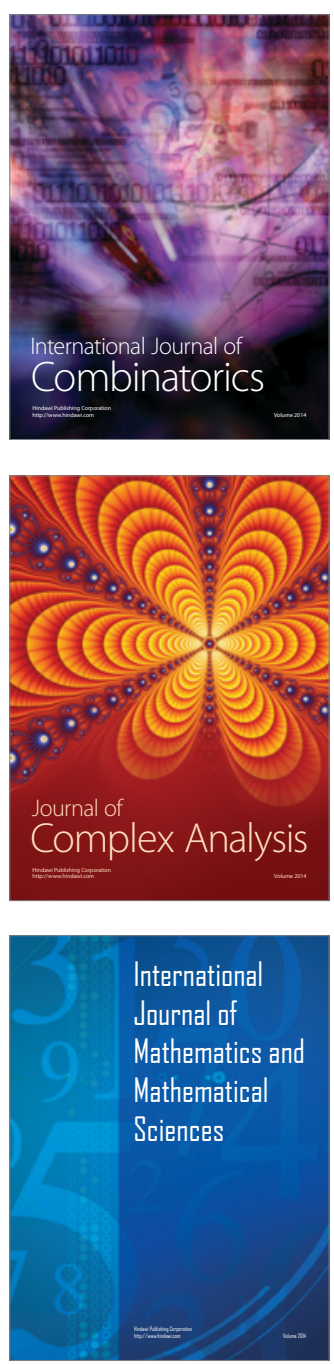
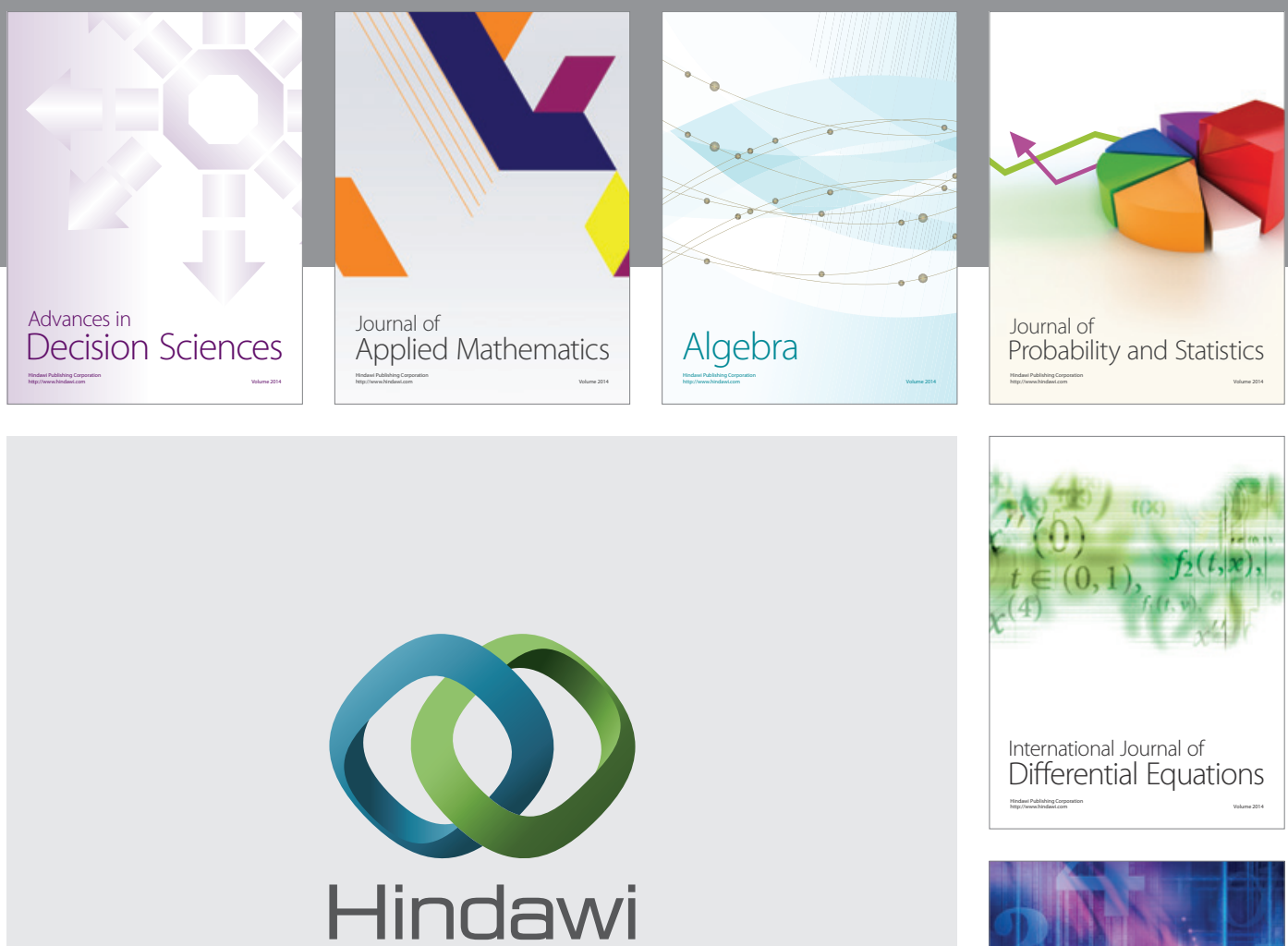

Submit your manuscripts at http://www.hindawi.com
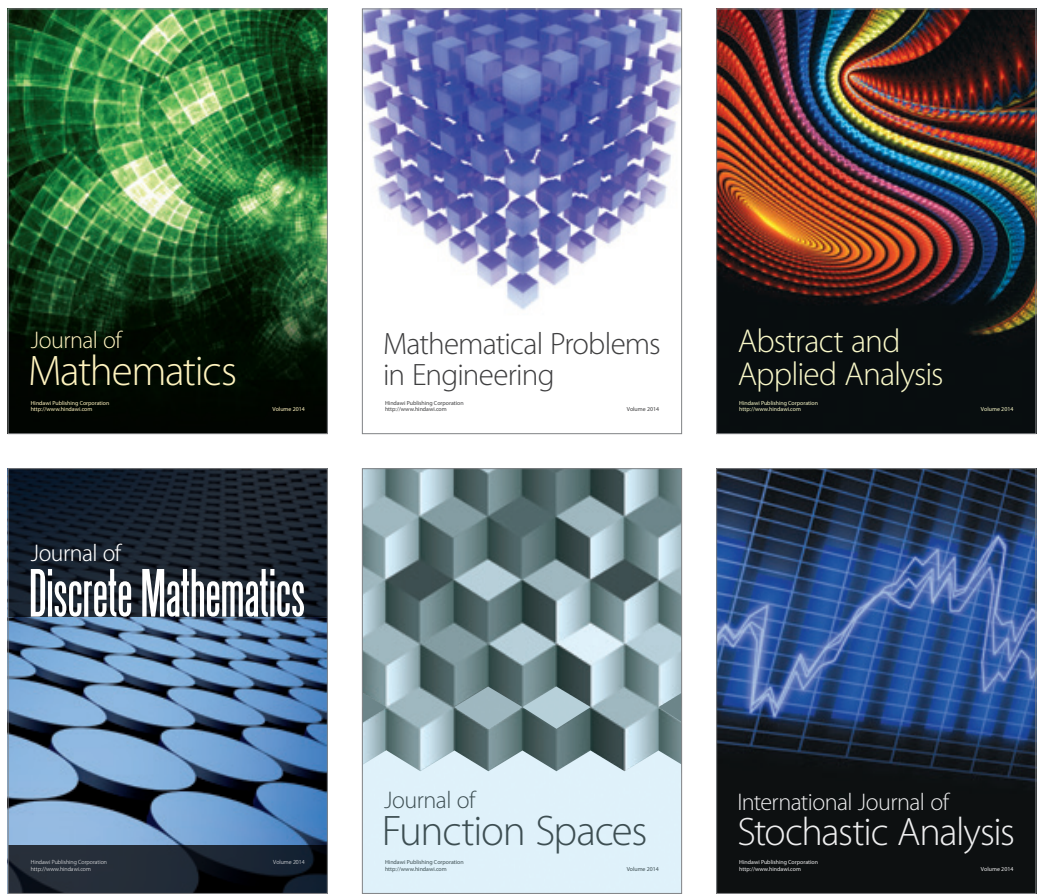

Journal of

Function Spaces

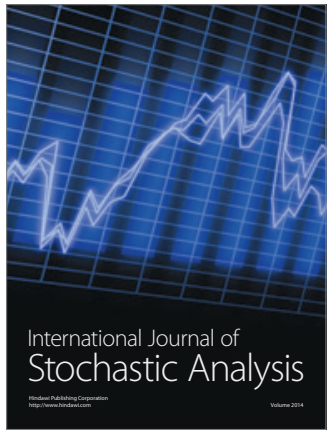

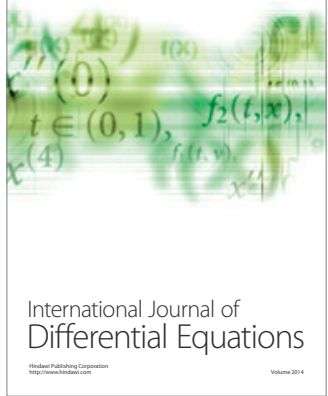
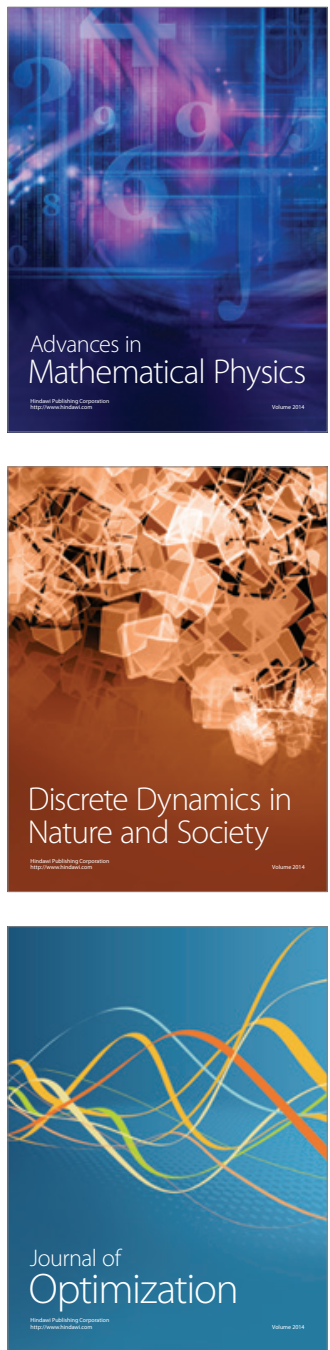